\title{
NORMED UNITS IN ABELIAN GROUP RINGS
}

\author{
PETER DANCHEV \\ Department of Mathematics, University of Plovdiv, Plovdiv 4000, Bulgaria BG \\ e-mail: peter_v@bulstrad.bg
}

(Received 15 December, 1999; accepted 2 February 2001)

\begin{abstract}
We compute explicitly the isomorphic structure of the normalized unit group of an abelian group ring under some minimal natural restrictions on the group basis and the coefficient ring. This enlarges affirmations due to ChatzidakisPappas (J. London Math. Soc., 1991) and Mollov (Publ. Math. Debrecen, 1971).
\end{abstract}

2000 Mathematics Subject Classification. 16U60, 16S34.

Introduction. Let $\mathrm{K}$ be an algebraically closed field of characteristic $\mathrm{p}\left(\mathrm{K}=\mathrm{K}^{-}\right.$, the algebraic closure of $\mathrm{K}$ ), let $\mathrm{F}$ be an arbitrary field of characteristic $\mathrm{p}$ and let $\mathrm{G}$ be an abelian group written multiplicatively. In this paper we completely describe the unit group $U F[G]$ of the group ring $F[G]$ when $G$ is a splitting sum of countable groups (in particular is a direct sum of cyclics). Our facts extend these established by Chatzidakis-Pappas in [1]. Further we provide the isomorphism type of UK[G] in the case that $\mathrm{G}$ is p-splitting. Our results generalize those obtained by Mollov [26, 27, 25] for $G$ torsion.

The present work is written in the sense of [1], but the technique used is (almost) algebraic. Its organization is as follows. In $\S 1$, we set up notation, terminology and some known results stated by us in [2-11]. In $\S 2$, we study the normed unit groups in abelian group rings. Here we state and prove our main theorems. We conclude in $\S 3$ with some questions left open and problems in the investigated theme.

1. Notation, conventions and previous results. Throughout $\mathrm{E}$ denotes an arbitrary field. We shall denote by $\mathrm{F}$ an arbitrary field of $\operatorname{char}(\mathrm{F})=\mathrm{p}$, by $\mathrm{K}$ an algebraically closed field of $\operatorname{char}(\mathrm{K})=\mathrm{p}\left(\mathrm{K}=\mathrm{K}^{-}\right.$, the algebraic cover of $\left.\mathrm{K}\right)$, and by $\mathrm{R}$ an abelian ring with 1 of $\operatorname{char}(R)=p$. For $G$ a commutative group and $p$ a prime integer, $G_{p}$ denotes the p-component of the torsion subgroup $G_{t}$ of $G$. As usual, $R[G]$ is the group ring with a group of normed invertible elements VR[G].

For $n$ a natural number, $\zeta_{n}$ will designate a primitive $n$-th root of unity. We let $\mu$ denote the group of all primitive $n$-th roots of unity for $n$ prime to $\operatorname{char}(F)=p$, and $\mu_{\mathrm{q}}$ the q-component of $\mu$. We let $\mathrm{F}^{*}=\mathrm{F} \backslash\{0\}$ designate the multiplicative group of $\mathrm{F}$ and more generally, we let UR be the multiplicative group of units of a ring R. For $\mathrm{H}$ a subgroup of $\mathrm{G}$ we define $\mathrm{I}(\mathrm{R}[\mathrm{G}] ; \mathrm{H})$ as the relative augmentation ideal of $\mathrm{R}[\mathrm{G}]$ with respect to $H$, generated by elements $1-h$, when $h$ varies in $H$. We define the set $1+\mathrm{I}(\mathrm{R}[\mathrm{G}] ; \mathrm{H})=\mathrm{V}(\mathrm{R}[\mathrm{G}] ; \mathrm{H})$.

Following the standard terminology, we say that G is $\Sigma$-cyclic (respectively $\Sigma$ countable) if $G$ is a direct sum of cyclic groups (a direct sum of countable groups, respectively). All other notations and terminology are in agreement with $[\mathbf{1 2}, \mathbf{1 4}, \mathbf{1 5}$. 
The next results are well documented [2-11], but for the convenience of the reader and for the sake of completeness we shall prove some of them, using a slightly different technique. The following are valid.

TheORem 1.1 [3-8]. Suppose that $\mathrm{G}$ is p-primary $\Sigma$-cyclic. Then $\mathrm{G}$ is a direct factor of VR[G] with a $\Sigma$-cyclic complement. Thus $\mathrm{VR}[\mathrm{G}]$ is $\Sigma$-cyclic. If $\mathrm{G}_{\mathrm{p}}$ is $\Sigma$ cyclic and $\mathrm{R}$ has a trivial nilradical, then $\mathrm{G}_{\mathrm{p}}$ is a direct factor of $\mathrm{SR}[\mathrm{G}]$ with a $\Sigma$-cyclic complementary factor. Thus $\mathrm{SR}[\mathrm{G}]$ is $\Sigma$-cyclic.

Theorem $1.2[\mathbf{2 4}, \mathbf{3}, \mathbf{6}]$. Suppose that $\mathrm{G}_{\mathrm{t}}$ is $\mathrm{p}$-torsion $\Sigma$-cyclic. Then $\mathrm{G}$ is a direct factor of $\mathrm{VF}[\mathrm{G}]$ with a $\Sigma$-cyclic complement. Thus if $\mathrm{G}$ is $\Sigma$-cyclic, then $\mathrm{VF}[\mathrm{G}]$ is $\Sigma$ cyclic and conversely.

Theorem 1.3 [5]. Suppose that $\mathrm{G}_{\mathrm{p}}$ has a countable limit length and $\mathrm{F}$ is perfect. Then $\mathrm{SF}[\mathrm{G}]$ is $\Sigma$-countable if and only if $\mathrm{G}_{\mathrm{p}}$ is $\Sigma$-countable. Moreover, if $\mathrm{G}_{\mathrm{p}}$ is $\Sigma$-countable, then $\mathrm{G}_{\mathrm{p}}$ is a direct factor of $\mathrm{SF}[\mathrm{G}]$ with a $\Sigma$-countable complement. If $\mathrm{G}_{\mathrm{t}}=\mathrm{G}_{\mathrm{p}}$, then $\mathrm{VF}[\mathrm{G}]$ is $\Sigma$-countable if and only if $\mathrm{G}$ is $\Sigma$-countable. Moreover, if $\mathrm{G}_{\mathrm{t}}$ is p-torsion $\Sigma$-countable, then $\mathrm{G}$ is a direct factor of $\mathrm{VF}[\mathrm{G}]$ with a $\Sigma$-countable complementary factor.

REMARK. Other facts in this direction of some interest and importance, the reader can find in our previous articles $[7,9,10,11]$.

2. Descriptions of $\mathbf{V F}[\mathbf{G}]$ and $\mathbf{V K}[\mathbf{G}]$. Some of the central theorems of this investigation were announced in [4]. We start this section with two simple observations. First, it is well known that $U R[G]=V R[G] \times U R$ and so the study of $U R[G]$ is reduced to that of $V R[G]$. Moreover, $U_{p} R[G]=V_{p} R[G] \times U_{p} R$, and the study of $\mathrm{U}_{\mathrm{p}} \mathrm{R}[\mathrm{G}]$ reduces to that of $\mathrm{V}_{\mathrm{p}} \mathrm{R}[\mathrm{G}]$. If $\mathrm{R}$ has a zero nilradical, then $\mathrm{U}_{\mathrm{p}} \mathrm{R}=1$ and $\mathrm{U}_{\mathrm{p}} \mathrm{R}[\mathrm{G}]=\mathrm{V}_{\mathrm{p}} \mathrm{R}[\mathrm{G}]$.

Next, we shall prove Theorems 1.1. and 1.2 in a more compact form.

THEOREM 2.1. (i) VR[G] is $\Sigma$-cyclic if and only if $\mathrm{G}$ is $\Sigma$-cyclic, provided that $\mathrm{G}$ is p-primary. If $\mathrm{G}$ is $\Sigma$-cyclic p-primary, then $\mathrm{G}$ is a direct factor of $\mathrm{VR}[\mathrm{G}]$.

(ii) $\mathrm{SR}[\mathrm{G}]$ is $\Sigma$-cyclic if and only if $\mathrm{G}_{\mathrm{p}}$ is $\Sigma$-cyclic, provided that $\mathrm{R}$ is without nilpotents. If $\mathrm{G}_{\mathrm{p}}$ is $\Sigma$-cyclic, then $\mathrm{G}_{\mathrm{p}}$ is a direct factor of $\mathrm{SR}[\mathrm{G}]$. Moreover, if $\mathrm{G}_{\mathrm{t}}$ is $\mathrm{p}$-torsion $\Sigma$-cyclic, then $\mathrm{G}$ is a direct factor of $\mathrm{VF}[\mathrm{G}]$ with a $\Sigma$-cyclic complement. Thus $\mathrm{VF}[\mathrm{G}]$ is $\Sigma$-cyclic if and only if $\mathrm{G}$ is $\Sigma$-cyclic, assuming that $\mathrm{G}_{\mathrm{t}}$ is $\mathrm{p}$ primary.

Proof. We have proved in [6], more generally, that if $\mathrm{H}$ is a $\Sigma$-cyclic p-group and if it is pure in $\mathrm{G}$, then $\mathrm{H}$ is a direct factor of the p-group $\mathrm{V}(\mathrm{R}[\mathrm{G}] ; \mathrm{H})$, where the last group is $\Sigma$-cyclic too. This is equivalent to $\mathrm{V}(\mathrm{R}[\mathrm{G}] ; \mathrm{H}) / \mathrm{H}$ being $\Sigma$-cyclic [12, p. 143, Theorem 28.2].

Let now, $\mathrm{H}=\mathrm{G}$. Consequently $\mathrm{V}(\mathrm{R}[\mathrm{G}] ; \mathrm{G})=\mathrm{VR}[\mathrm{G}]$. Moreover if $\mathrm{H}=\mathrm{G}_{\mathrm{p}}$ and the nilradical of $R$ is zero, then $V\left(R[G] ; G_{p}\right)=S R[G]$. (See $[3,5]$.) That is why (i) and the first half of (ii) hold.

Suppose that $G_{t}=G_{p}$. Therefore $V F[G] / S F[G] \cong V F\left[G / G_{p}\right]=G / G_{p}$, by virtue of the classical result of G. Higman (cf. [14]). Then $V F[G]=G S F[G]$, where 
$\mathrm{G} \cap \mathrm{SF}[\mathrm{G}]=\mathrm{G}_{\mathrm{p}}$, and besides $\mathrm{VF}[\mathrm{G}] / \mathrm{G} \cong \mathrm{SF}[\mathrm{G}] / \mathrm{G}_{\mathrm{p}}$. From the above claim part (ii) follows.

REMARK. T. Mollov [25] has proved the first statement of (i), but the claim about the direct factor was not discussed.

Moreover, W. May [22] proved that if $\mathrm{G}$ is a $\Sigma$-countable p-group and $\mathrm{R}$ is perfect, then $\mathrm{G}$ is a direct factor of VR[G] with a $\Sigma$-countable complement. Thus $\mathrm{VR}[\mathrm{G}]$ is $\Sigma$-countable. If $\mathrm{G}$ has countable length and $\mathrm{VR}[\mathrm{G}]$ is a $\Sigma$-countable group, then $\mathrm{G}$ is $\Sigma$-countable for R arbitrary (i.e. it need not be perfect). In this light, May in [23] showed that the simply presented p-group $G$ is a direct factor of VF[G] with a simply presented complement provided that $F$ is perfect. Moreover in [24] he proved the assertions about the direct factor (ii), for $R$ a field and $G_{t}=G_{p}$ (his idea and technique are differ from ours). Important facts which enlarge the above mentioned affirmations are established by us in $[\mathbf{1 0}, \mathbf{1 1}]$.

Next, we generalize in some aspect the theorems above. (See [11], too.)

Claim 2.1. Assume that $\mathrm{F}$ is perfect and $\mathrm{G}$ is p-splitting. Then $\mathrm{SF}[\mathrm{G}]$ is simply presented (in particular $\Sigma$-countable) if and only if $\mathrm{G}_{\mathrm{p}}$ is.

Proof. Write $\mathrm{G}=\mathrm{G}_{\mathrm{p}} \times \mathrm{M}$. Then clearly $\mathrm{SF}\left[\mathrm{G}_{\mathrm{p}}\right]$ is a direct factor of $\mathrm{SF}[\mathrm{G}]$ and hence $S F[G]$ simply presented yields the same for $S F\left[G_{p}\right]$; i.e. $G_{p}$ is simply presented $[23,14]$.

Now we treat the more difficult converse question. Select a smooth ascending chain $1=\mathrm{N}_{0} \subseteq \ldots \subseteq \mathrm{N}_{\alpha} \subseteq \ldots(\alpha<\mu)$ of nice subgroups of $\mathrm{G}_{\mathrm{p}}=\bigcup_{\alpha<\mu} \mathrm{N}_{\alpha}$ (and hence of G) such that $\left|N_{\alpha+1} / N_{\alpha}\right| \leq \aleph_{0}$ whenever $\alpha+1<\mu$. But SF[G] $=V\left(F[G] ; G_{p}\right)$ by $[\mathbf{3 , 5}]$ and therefore there is a smooth ascending sequence

$$
1=\mathrm{V}\left(\mathrm{F}[\mathrm{G}] ; \quad \mathrm{N}_{0}\right) \subseteq \ldots \subseteq \mathrm{V}\left(\mathrm{F}[\mathrm{G}] ; \mathrm{N}_{\alpha}\right) \subseteq \ldots
$$

of nice subgroups of SF[G] (see [23]) with $\mathrm{SF}[\mathrm{G}]=\bigcup_{\alpha<\mu} \mathrm{V}\left(\mathrm{F}[\mathrm{G}] ; \mathrm{N}_{\alpha}\right.$ ). Adapting the technique described on page 407 of [23], we may obtain a nice composition series for $\mathrm{SF}[\mathrm{G}]$ that verifies that $\mathrm{SF}[\mathrm{G}]$ is simply presented. But length $\mathrm{SF}[\mathrm{G}]=$ length $\mathrm{G}_{\mathrm{p}}(\leq \Omega$ for the $\Sigma$-countable case [12]), which finishes the proof in general.

The following two formulae are necessary for our presentation and are given in a slightly different form from the original.

Theorem (Chatzidakis-Pappas [1]). Let $\mathrm{E}$ be a field and let $\mathrm{G}$ be an abelian group with no element of order char(E).

$$
\begin{aligned}
& \text { If } \mathrm{G} \text { is infinite torsion, then } \mathrm{UE}^{-}[\mathrm{G}] \cong \mathrm{X}_{|\mathrm{G}|} \mathrm{E}^{-*} \text {. } \\
& \text { If } \mathrm{G} \text { splits and } \mathrm{G}_{\mathrm{t}} \text { is infinite, then } \mathrm{UE}[\mathrm{G}] \cong \mathrm{UE}\left[\mathrm{G}_{\mathrm{t}}\right] \times\left(\times_{|\mathrm{Gt}|} \mathrm{G} / \mathrm{G}_{\mathrm{t}}\right) \text {. }
\end{aligned}
$$

In their paper [1], Chatzidakis and Pappas completely describe UE[G] when G is torsion $\Sigma$-countable without elements of order char(E). They have demonstrated that $U E[G]$ is isomorphic to a direct sum of multiplicative groups of cyclotomic extensions of $E$ and computed explicitly their exponents. In particular, when $G$ is an infinite torsion $\Sigma$-cyclic group, the following is valid. 
$\mathrm{UE}[\mathrm{G}] \cong \prod_{d=0}^{\infty} \times_{l_{\mathrm{d}}} \mathrm{E}\left(\zeta_{\mathrm{d}}\right)^{*}$, where $1_{\mathrm{d}}=\left|\left\{\mathrm{g} \in \mathrm{G}_{\mathrm{t}} \mid \operatorname{order}(\mathrm{g})=\mathrm{d}\right\}\right| /\left(\mathrm{E}\left(\zeta_{\mathrm{d}}\right): \mathrm{E}\right)$.

However, it is well known and documented that if $\mathrm{G}$ is finite, then (see [37] or [1])

$$
\mathrm{UE}[\mathrm{G}] \cong \prod_{\mathrm{d} / / \mathrm{G} \mid} \times_{\mathrm{ld}} \mathrm{E}\left(\zeta_{\mathrm{d}}\right)^{*}
$$

Further we shall establish some supplements and expansions of the last theorem. For this we first need a few preliminaries.

Lemma 2.1. Suppose that $1 \in \mathrm{L} \leq \mathrm{R}, \mathrm{B} \leq \mathrm{G}, \mathrm{X} \leq \mathrm{G}$. Then

$$
\mathrm{V}(\mathrm{R}[\mathrm{G}] ; \mathrm{B}) \cap \mathrm{V}(\mathrm{L}[\mathrm{X}] ; \mathrm{X})=\mathrm{V}(\mathrm{L}[\mathrm{X}] ; \mathrm{X} \cap \mathrm{B}) .
$$

Proof. Given $\mathrm{y}$ in the left-hand side, we have $\mathrm{y}=\sum_{\mathrm{x} \in \mathrm{X}} \mathrm{f}_{\mathrm{x}} \mathrm{x}$ and

$$
\sum_{x \in g B} f_{x}=\left\{\begin{array}{ll}
0 & g \notin B \\
1 & g \in B
\end{array}, \text { for each } g \in G\right.
$$

where $f_{x} \in$ L. Choose arbitrary $a \in X$. Furthermore $a B \cap X=a(B \cap X)$ and moreover we get

$$
\sum_{x \in a B \cap X} f_{x}=\sum_{x \in a(B \cap X)} f_{x}=\left\{\begin{array}{ll}
0 & a \notin B \cap X \\
1 & a \in B \cap X
\end{array} .\right.
$$

Finally, $y \in \mathrm{V}(\mathrm{L}[\mathrm{X}] ; \mathrm{X} \cap \mathrm{B})$, as required.

Now we are in a position to formulate and prove the following two results.

Lemma 2.2. If $\mathrm{G}=\mathrm{G}_{\mathrm{p}} \times \mathrm{M}$, then

$$
\begin{gathered}
\mathrm{VR}[\mathrm{G}]=\mathrm{VR}[\mathrm{M}] \times \mathrm{V}\left(\mathrm{R}[\mathrm{G}] ; \mathrm{G}_{\mathrm{p}}\right), \\
\mathrm{VF}[\mathrm{G}]=\mathrm{VF}[\mathrm{M}] \times \mathrm{SF}[\mathrm{G}], \\
\mathrm{UF}[\mathrm{G}]=\mathrm{UF}[\mathrm{M}] \times \mathrm{SF}[\mathrm{G}] .
\end{gathered}
$$

Proof. Because $\mathrm{R}[\mathrm{G}]=\mathrm{R}[\mathrm{M}]\left[\mathrm{G}_{\mathrm{p}}\right]$, for each $\mathrm{x} \in \mathrm{VR}[\mathrm{G}]$ we have $\mathrm{x}=\sum_{\mathrm{a} \in \mathrm{G}_{\mathrm{p}}} \mathrm{x}_{\mathrm{a}} \mathrm{a}$, where $\mathrm{x}_{\mathrm{a}} \in \mathrm{R}[\mathrm{M}]$. Set $x^{-}=\sum_{\mathrm{a} \in \mathrm{G}_{\mathrm{p}}} \mathrm{x}_{\mathrm{a}}$. Consequently $\mathrm{x}=\mathrm{x}^{-}+\sum_{\mathrm{a} \in \mathrm{G}_{\mathrm{p}}} \mathrm{x}_{\mathrm{a}} \cdot(\mathrm{a}-1)$. Apparently $\mathrm{x}^{\mathrm{p}^{\mathrm{k}}}=\mathrm{x}^{-\mathrm{p}^{\mathrm{k}}}$ for any natural $\mathrm{k}$. But $\mathrm{x}^{\mathrm{p}^{\mathrm{k}}} \in \mathrm{VR}[\mathrm{G}]$ and so $\mathrm{x}^{-} \in \mathrm{VR}[\mathrm{G}]$. Hence $x^{-} \in V R[G] \cap R[M]=V R[M]$. Writing $v=1+x^{-(-1)} \cdot \sum_{a \in G_{p} \backslash\{1\}} x_{a} \cdot(a-1)$, we easily deduce that $\mathrm{x}=\mathrm{x}^{-} \mathrm{v}$, where $\mathrm{v} \in \mathrm{V}\left(\mathrm{R}[\mathrm{G}] ; \mathrm{G}_{\mathrm{p}}\right)$. It is clear that, $\mathrm{VR}[\mathrm{G}] \subseteq \mathrm{VR}[\mathrm{M}] . \mathrm{V}\left(\mathrm{R}[\mathrm{G}] ; \mathrm{G}_{\mathrm{p}}\right)$. Certainly by an application of Lemma 2.1, $\mathrm{VR}[\mathrm{M}] \cap \mathrm{V}\left(\mathrm{R}[\mathrm{G}] ; \mathrm{G}_{\mathrm{p}}\right) \subseteq \mathrm{V}\left(\mathrm{R}[\mathrm{M}] ; \mathrm{M} \cap \mathrm{G}_{\mathrm{p}}\right)=1$, which completes the proof of (5).

The other two equalities hold by the formulae $S F[G]=V\left(F[G] ; G_{p}\right)$ (cf. [5]) and $\mathrm{UF}[\mathrm{G}]=\mathrm{VF}[\mathrm{G}] \times \mathrm{F}^{*}$. 
The next result is important. Its proof is obvious and we omit the details.

Lemma 2.3. Suppose $\mathrm{R}_{\mathrm{i}}$ are commutative rings with identities $(\mathrm{i}=1, \ldots, \mathrm{j})$. Then $\left(\oplus_{\mathrm{i}} \mathrm{R}_{\mathrm{i}}\right)[\mathrm{G}] \cong \oplus_{\mathrm{i}} \mathrm{R}_{\mathrm{i}}[\mathrm{G}]$.

Now we can state our main result.

THEOREM 2.2. (a) Let $\mathrm{E}$ be a field and let $\mathrm{G}$ be an abelian group without elements of order char(E).

$$
\begin{gathered}
\mathrm{UE}^{-}[\mathrm{G}] \cong\left(X_{\left|\mathrm{G}_{\mathrm{t}}\right|} \mathrm{G} / \mathrm{G}_{\mathrm{t}}\right) \times\left(\mathrm{X}_{\left|\mathrm{G}_{\mathrm{t}}\right|} \mathrm{E}^{-*}\right) \\
\mathrm{UE}^{-}[\mathrm{G}] \cong\left(\mathrm{X}_{\left|\mathrm{G}_{\mathrm{t}}\right|} \mathrm{G} / \mathrm{G}_{\mathrm{t}}\right) \times\left(\mathrm{X}_{\left|\mathrm{G}_{\mathrm{t}}\right|} \mathrm{Q} / \mathrm{Z}\right) \times\left(\mathrm{X}_{\left|\mathrm{G}_{\mathrm{t}}\right|} \mathrm{X}_{\left|\mathrm{E}^{-}\right|} \mathrm{Q}\right),
\end{gathered}
$$

if $\operatorname{char}(\mathrm{E})=0$.

$$
\mathrm{UE}^{-}[\mathrm{G}] \cong\left(\mathrm{X}_{\left|\mathrm{G}_{\mathrm{t}}\right|} \mathrm{G} / \mathrm{G}_{\mathrm{t}}\right) \times\left(\mathrm{X}_{\left|\mathrm{G}_{\mathrm{t}}\right|} \mathrm{X}_{\mathrm{q} \neq \mathrm{p}} \mathrm{Z}\left(\mathrm{q}^{\infty}\right)\right) \times\left(\mathrm{X}_{\left|\mathrm{G}_{\mathrm{t}}\right|} \mathrm{X}_{\eta} \mathrm{Q}\right),
$$

if $\operatorname{char}(\mathrm{E})=\mathrm{p} \neq 0$, where $\eta=0$ or $\eta=\left|\mathrm{E}^{-}\right|$.

(b) Suppose that $\mathrm{G}$ is p-splitting.

$$
\begin{aligned}
\mathrm{UK}[\mathrm{G}] & \cong\left(\mathrm{X}_{\left|\mathrm{G}_{\mathrm{t}} / \mathrm{G}_{\mathrm{p}}\right|} \mathrm{G} / \mathrm{G}_{\mathrm{t}}\right) \times\left(\mathrm{X}_{\left|\mathrm{G}_{\mathrm{t}} / \mathrm{G}_{\mathrm{p}}\right|} \mathrm{K}^{*}\right) \times \mathrm{SK}[\mathrm{G}] . \\
\mathrm{UK}[\mathrm{G}] & \cong\left(X_{\left|\mathrm{G}_{\mathrm{t}} / \mathrm{G}_{\mathrm{p}}\right|} \mathrm{G} / \mathrm{G}_{\mathrm{t}}\right) \times\left(\mathrm{X}_{\left|\mathrm{G}_{\mathrm{t}} / \mathrm{G}_{\mathrm{p}}\right|} \mathrm{X}_{\mathrm{q} \neq \mathrm{p}} \mathrm{Z}\left(\mathrm{q}^{\infty}\right)\right) \\
& \times\left(\mathrm{X}_{\left|\mathrm{G}_{\mathrm{t}} / \mathrm{G}_{\mathrm{p}}\right|} \mathrm{X}_{\eta} \mathrm{Q}\right) \times \mathrm{SK}[\mathrm{G}], \text { where } \eta=0 \text { or } \eta=|\mathrm{K}| .
\end{aligned}
$$

(c) Assume $\mathrm{G}_{\mathrm{t}}$ is finite and $\mathrm{E}$ is a field. If char $(\mathrm{E})=0$, then we have

$$
\mathrm{UE}[\mathrm{G}] \cong\left(X_{\alpha} \mathrm{G} / \mathrm{G}_{\mathrm{t}}\right) \times\left(\prod_{\mathrm{d} /\left|\mathrm{G}_{\mathrm{t}}\right|} \mathrm{X}_{\mathrm{l}_{\mathrm{d}}} \mathrm{E}\left(\zeta_{\mathrm{d}}\right)^{*}\right), \quad \alpha=\sum_{\mathrm{d} /\left|\mathrm{G}_{\mathrm{t}}\right|} 1_{\mathrm{d}}
$$

and if $\operatorname{char}(\mathrm{E})=\mathrm{p}>0$, then we have

$$
\begin{gathered}
\mathrm{UE}[\mathrm{G}] \cong\left(X_{\beta} \mathrm{G} / \mathrm{G}_{\mathrm{t}}\right) \times\left(\prod_{\mathrm{d} /\left|\mathrm{G}_{\mathrm{t}} / \mathrm{G}_{\mathrm{p}}\right|} \mathrm{X}_{\mathrm{m}_{\mathrm{d}}} \mathrm{E}\left(\zeta_{\mathrm{d}}\right)^{*}\right) \times \mathrm{SE}[\mathrm{G}], \\
\beta=\sum_{\mathrm{d} /\left|\mathrm{G}_{\mathrm{t}} / \mathrm{G}_{\mathrm{p}}\right|} \mathrm{m}_{\mathrm{d}},
\end{gathered}
$$

where $1_{\mathrm{d}}=\left|\left\{\mathrm{g} \in \mathrm{G}_{\mathrm{t}} \mid \operatorname{order}(\mathrm{g})=\mathrm{d}\right\}\right| /\left(\mathrm{E}\left(\zeta_{\mathrm{d}}\right): \mathrm{E}\right) ; \quad \sum_{\mathrm{d} /\left|\mathrm{G}_{\mathrm{t}}\right|} 1_{\mathrm{d}} \cdot\left(\mathrm{E}\left(\zeta_{\mathrm{d}}\right): \mathrm{E}\right)=\left|\mathrm{G}_{\mathrm{t}}\right|$, and $\mathrm{m}_{\mathrm{d}}=\left|\left\{\mathrm{g} \in \mathrm{G}_{\mathrm{t}} / \mathrm{G}_{\mathrm{p}} \mid \operatorname{order}(\mathrm{g})=\mathrm{d}\right\}\right| /\left(\mathrm{E}\left(\zeta_{\mathrm{d}}\right): \mathrm{E}\right) ; \quad \sum_{\mathrm{d} /\left|\mathrm{G}_{\mathrm{t}} / \mathrm{G}_{\mathrm{p}}\right|} \mathrm{m}_{\mathrm{d}} \cdot\left(\mathrm{E}\left(\zeta_{\mathrm{d}}\right): \mathrm{E}\right)=\left|\mathrm{G}_{\mathrm{t}} / \mathrm{G}_{\mathrm{p}}\right|$. Moreover, $\mathrm{SE}[\mathrm{G}]$ is bounded and the Ulm-Kaplansky functions [28] serve to classify $\mathrm{SE}[\mathrm{G}]$.

(d) Suppose that $\mathrm{G}$ splits and $\mathrm{E}$ is a field. If $\operatorname{char}(\mathrm{E})=0$, then

$$
\mathrm{UE}[\mathrm{G}] \cong \mathrm{UE}\left[\mathrm{G}_{\mathrm{t}}\right] \times\left(\mathrm{X}_{\alpha} \mathrm{G} / \mathrm{G}_{\mathrm{t}}\right)
$$

where $\alpha=\left|\mathrm{G}_{\mathrm{t}}\right| \geq \aleph_{0} \quad$ or $\quad \alpha=\sum_{\mathrm{d} /\left|\mathrm{G}_{\mathrm{t}}\right|} 1_{\mathrm{d}}$ when $\left|\mathrm{G}_{\mathrm{t}}\right|<\aleph_{0}$, where $1_{\mathrm{d}}=\mid\left\{\mathrm{g} \in \mathrm{G}_{\mathrm{t}} \mid\right.$ $\operatorname{order}(\mathrm{g})=\mathrm{d}\} \mid /\left(\mathrm{E}\left(\zeta_{\mathrm{d}}\right): \mathrm{E}\right)$, and if $\operatorname{char}(\mathrm{E})=\mathrm{p}>0$ then we have 


$$
\mathrm{UE}[\mathrm{G}] \cong \mathrm{UE}\left[\mathrm{G}_{\mathrm{t}} / \mathrm{G}_{\mathrm{p}}\right] \times\left(\mathrm{X}_{\beta} \mathrm{G} / \mathrm{G}_{\mathrm{t}}\right) \times \mathrm{SE}[\mathrm{G}],
$$

where $\beta=\left|\mathrm{G}_{\mathrm{t}} / \mathrm{G}_{\mathrm{p}}\right| \geq \aleph_{0} \quad$ or $\quad \beta=\sum_{\mathrm{d} /\left|\mathrm{G}_{\mathrm{t}} / \mathrm{G}_{\mathrm{p}}\right|} \mathrm{m}_{\mathrm{d}} \quad$ when $\quad\left|\mathrm{G}_{\mathrm{t}} / \mathrm{G}_{\mathrm{p}}\right|<\aleph_{0}$; here $\mathrm{m}_{\mathrm{d}}=\left|\left\{\mathrm{g} \in \mathrm{G}_{\mathrm{t}} / \mathrm{G}_{\mathrm{p}} \mid \operatorname{order}(\mathrm{g})=\mathrm{d}\right\}\right| /\left(\mathrm{E}\left(\zeta_{\mathrm{d}}\right): \mathrm{E}\right)$.

(e) Let $\mathrm{G}$ be splitting such that $\mathrm{G}_{\mathrm{t}} / \mathrm{G}_{\mathrm{p}}$ is $\Sigma$-cyclic.

$$
\begin{aligned}
& \mathrm{UF}[\mathrm{G}] \cong\left(X_{\left|\mathrm{G}_{\mathrm{t}} / \mathrm{G}_{\mathrm{p}}\right|} \mathrm{G} / \mathrm{G}_{\mathrm{t}}\right) \times\left(\prod_{\mathrm{n}=0}^{\infty} \times \mathrm{m}_{\mathrm{n}} \mathrm{F}\left(\zeta_{\mathrm{n}}\right)^{*}\right) \times \mathrm{SF}[\mathrm{G}], \quad\left|\mathrm{G}_{\mathrm{t}} / \mathrm{G}_{\mathrm{p}}\right| \geq \aleph_{0} . \\
& \mathrm{UF}[\mathrm{G}] \cong\left(\times_{\gamma} \mathrm{G} / \mathrm{G}_{\mathrm{t}}\right) \times\left(\prod_{\mathrm{n} /\left|\mathrm{G}_{\mathrm{t}} / \mathrm{G}_{\mathrm{p}}\right|} \times \mathrm{m}_{\mathrm{n}} \mathrm{F}\left(\zeta_{\mathrm{n}}\right)^{*}\right) \times \mathrm{SF}[\mathrm{G}], \quad\left|\mathrm{G}_{\mathrm{t}} / \mathrm{G}_{\mathrm{p}}\right|<\aleph_{0},
\end{aligned}
$$

where $\gamma=\sum_{\mathrm{n} /\left|\mathrm{G}_{\mathrm{t}} / \mathrm{G}_{\mathrm{p}}\right|} \mathrm{m}_{\mathrm{n}}$ and $\mathrm{m}_{\mathrm{n}}=\left|\left\{\mathrm{g} \in \mathrm{G}_{\mathrm{t}} / \mathrm{G}_{\mathrm{p}} \mid \operatorname{order}(\mathrm{g})=\mathrm{n}\right\}\right| /\left(\mathrm{E}\left(\zeta_{\mathrm{n}}\right): \mathrm{E}\right)$. If $\mathrm{G}_{\mathrm{p}}$ is $\Sigma$-cyclic, then $\mathrm{SF}[\mathrm{G}]$ is $\Sigma$-cyclic and thus the Ulm-Kaplansky cardinal invariants [28] serve to classify $\mathrm{SF}[\mathrm{G}]$.

(f) Let $\mathrm{G}$ be splitting so that $\mathrm{G}_{\mathrm{t}} / \mathrm{G}_{\mathrm{p}}$ is $\Sigma$-countable. Besides let $\mathrm{D}_{\mathrm{q}}$ be the maximal divisible subgroup of $\mathrm{G}_{\mathrm{q}}$ and $\mathrm{D}=\mathrm{X}_{\mathrm{q} \neq \mathrm{p}} \mathrm{D}_{\mathrm{q}}$, where $\mathrm{q}$ is a prime. Let $\mathrm{P}$ be the set of all primes $\mathrm{q} \neq \mathrm{p}$. For every finite $\mathrm{T} \subset \mathrm{P}$ and every integer $\mathrm{n}$ relatively prime to the members of $\mathrm{T}$ (following Chatzidakis and Pappas), we define a cardinal number $\mathrm{m}(\mathrm{T}, \mathrm{n})$ as follows:

- $\mathrm{m}(\varnothing, 1)=1$;

- $\mathrm{G}_{\mathrm{q}}^{\mathrm{q}^{\omega}} \cong 1, \mathrm{~m}(\{\mathrm{q}\}, 1)=\left|\mathrm{G}_{\mathrm{q}}\right|$; otherwise $\mathrm{m}(\{\mathrm{q}\}, 1)=0$;

- $\mathrm{m}(\mathrm{T}, 1)=\prod_{\mathrm{q} \in \mathrm{T}} \mathrm{m}(\{\mathrm{q}\}, 1)$;

- $\mathrm{m}(\mathrm{T}, \mathrm{n})=\mathrm{m}(\mathrm{T}, 1) \alpha_{n}$, where $\alpha_{\mathrm{n}}\left(\mathrm{F}\left(\zeta_{\mathrm{n}}\right): \mathrm{F}\right)=\mid\left\{\mathrm{a} \in\left(\mathrm{X}_{\mathrm{q} \neq \mathrm{p}} \mathrm{G}_{\mathrm{q}}\right) / \mathrm{D} \mid\right.$ a has order $\left.\mathrm{n}\right\} \mid$. The following properties hold.

$$
\mathrm{UF}[\mathrm{G}] \cong\left(\mathrm{X}_{\left|\mathrm{G}_{\mathrm{t}} / \mathrm{G}_{\mathrm{p}}\right|} \mathrm{G} / \mathrm{G}_{\mathrm{t}}\right) \times\left(\prod_{\mathrm{T}, \mathrm{n}} \mathrm{X}_{\mathrm{m}(\mathrm{T}, \mathrm{n})} \mathrm{F}\left(\zeta_{\mathrm{n}}, \mu_{\mathrm{q}}\right)^{*}\right) \times \mathrm{SF}[\mathrm{G}]
$$

where $\left|\mathrm{G}_{\mathrm{t}} / \mathrm{G}_{\mathrm{p}}\right| \geq \aleph_{0}$, or

$$
\mathrm{UF}[\mathrm{G}] \cong\left(X_{\gamma} \mathrm{G} / \mathrm{G}_{\mathrm{t}}\right) \times\left(\prod_{\mathrm{n} /\left|\mathrm{G}_{\mathrm{t}} / \mathrm{G}_{\mathrm{p}}\right|} \mathrm{X}_{\mathrm{m}_{\mathrm{n}}} \mathrm{F}\left(\zeta_{\mathrm{n}}\right)^{*}\right) \times \mathrm{SF}[\mathrm{G}],
$$

where $\left|\mathrm{G}_{\mathrm{t}} / \mathrm{G}_{\mathrm{p}}\right|<\aleph_{0}$. Moreover if $\mathrm{G}_{\mathrm{p}}$ is $\Sigma$-countable and $\mathrm{F}$ is perfect, then $\mathrm{SF}[\mathrm{G}]$ is $\Sigma$ countable and thus the Ulm-Kaplansky cardinal functions [28] serve to classify $\mathrm{SF}[\mathrm{G}]$.

Proof. (a) We shall distinguish two basic cases.

Case $1-\mathrm{G}$ splitting. If $\left|\mathrm{G}_{\mathrm{t}}\right| \geq \aleph_{0}$, then the isomorphism holds by application of (1) and (2). Now, assume that $\left|G_{t}\right|<\aleph_{0}$. Hence $E^{-}\left[G_{t}\right] \cong \oplus_{\left|G_{t}\right|} E^{-}$, and $\mathrm{E}^{-}[\mathrm{G}]=\mathrm{E}^{-}\left[\mathrm{G}_{\mathrm{t}}\right]\left[\mathrm{G} / \mathrm{G}_{\mathrm{t}}\right]$. Consequently, by Lemma $2.3, \mathrm{E}^{-}[\mathrm{G}] \cong \oplus_{\left|G_{t}\right|} \mathrm{E}^{-}\left[\mathrm{G} / \mathrm{G}_{\mathrm{t}}\right]$ and immediately we deduce that $\mathrm{UE}^{-}[\mathrm{G}] \cong \mathrm{X}_{\left|\mathrm{G}_{\mathrm{t}}\right|} \mathrm{UE}^{-}\left[\mathrm{G} / \mathrm{G}_{\mathrm{t}}\right]=\mathrm{X}_{\left|\mathrm{G}_{\mathrm{t}}\right|}\left(\mathrm{G} / \mathrm{G}_{\mathrm{t}} \times \mathrm{E}^{-*}\right)$ according to the classical Higman's result (cf. [14]), completing the proof in this case.

Case 2- $\mathrm{G}$ arbitrary. Select $\mathrm{H}=\mathrm{G}_{\mathrm{t}} \times \mathrm{G} / \mathrm{G}_{\mathrm{t}}$. By [19], $\mathrm{E}^{-}[\mathrm{H}] \cong \mathrm{E}^{-}[\mathrm{G}]$, whence $\mathrm{UE}^{-}[\mathrm{H}] \cong \mathrm{UE}^{-}[\mathrm{G}]$. From Case 1 it follows that $\mathrm{UE}^{-}[\mathrm{H}] \cong\left(\mathrm{X}_{\left|G_{t}\right|} G / G_{t}\right) \times$ $\left(\mathrm{X}_{\left|G_{t}\right|} E^{-*}\right)$, and so we are done.

It is not difficult to see that $\mathrm{E}^{-*}$ is divisible. From the monograph [12, p. 369, Theorem 127.3] (see also the first book of [12]) it follows that $\mathrm{E}^{-*} \cong \mathrm{Q} / \mathrm{Z} \times \mathrm{X}_{\left|\mathrm{E}^{-}\right|} \mathrm{Q}$ 
for $\operatorname{char}(\mathrm{E})=0$, and $\mathrm{E}^{-*} \cong \mathrm{X}_{\mathrm{q} \neq \mathrm{p}} \mathrm{Z}\left(\mathrm{q}^{\infty}\right) \times \mathrm{X}_{\eta} \mathrm{Q}$ for $\operatorname{char}(\mathrm{E})=\mathrm{p} \neq 0$, where $\mathrm{q}$ is a prime, $\eta$ is a cardinal $\left(\eta=0\right.$ if $E$ is an algebraic extension of finite field $\Longleftrightarrow E^{-*}$ is torsion; or $\eta=\left|\mathrm{E}^{-}\right|$otherwise) and $\mathrm{Q}$ is an additive group of rationals. That is why for the above condition on $G$, the dependences (9) and (10) are true. Thus (a) is proved.

(b) Since $G$ p-splits, $G \cong G_{p} \times G / G_{p}$. Thus, by (7), UK $[G] \cong U K\left[G / G_{p}\right] \times$ SK[G]. Finally we need only apply the formulas (8) and (10). This completes the proof of (b).

(c) The torsion part $G_{t}$ is finite and hence bounded. However it is pure in $G$ and therefore $G$ splits; i.e. $G \cong G_{t} \times G / G_{t}$ (see [12, p. 140, Theorem 27.5] (L. Kulikov). Thus $\mathrm{E}[\mathrm{G}] \cong \mathrm{E}\left[\mathrm{G}_{\mathrm{t}}\right]\left[\mathrm{G} / \mathrm{G}_{\mathrm{t}}\right]$. We shall assume that char(E) does not divide $\left|\mathrm{G}_{\mathrm{t}}\right|$ (so that $\operatorname{char}(E)=0$ or $\operatorname{char}(E)=p \neq 0$ when $\left.G_{p}=1\right)$. That is why employing the classical Maschke's criterion, $\mathrm{E}\left[\mathrm{G}_{\mathrm{t}}\right]$ is a semisimple group algebra [13], and moreover it is Artinian. Hence, by $[37,13]$, we have $E\left[G_{t}\right] \cong \sum_{d /\left|G_{t}\right|} \oplus 1_{d} E\left(\zeta_{d}\right)$ and $\sum_{\mathrm{d} /\left|\mathrm{G}_{\mathrm{t}}\right|} 1_{\mathrm{d}} \cdot\left(\mathrm{E}\left(\zeta_{\mathrm{d}}\right): \mathrm{E}\right)=\left|\mathrm{G}_{\mathrm{t}}\right|$, where $1_{\mathrm{d}}$ are calculated as above.

Furthermore $\mathrm{E}[\mathrm{G}] \cong \sum_{\mathrm{d} /\left|\mathrm{G}_{\mathrm{t}}\right|} \oplus 1_{\mathrm{d}} \mathrm{E}\left(\zeta_{\mathrm{d}}\right)\left[\mathrm{G} / \mathrm{G}_{\mathrm{t}}\right]$, using Lemma 2.3.

Apparently $\quad \mathrm{UE}[\mathrm{G}] \cong \prod_{\mathrm{d} /\left|\mathrm{G}_{\mathrm{t}}\right|} \times_{\mathrm{l}_{\mathrm{d}}} \mathrm{UE}\left(\zeta_{\mathrm{d}}\right)\left[\mathrm{G} / \mathrm{G}_{\mathrm{t}}\right]=\prod_{\mathrm{d} /\left|\mathrm{G}_{\mathrm{t}}\right|} \times_{\mathrm{l}_{\mathrm{d}}}\left[\mathrm{G} / \mathrm{G}_{\mathrm{t}} \times \mathrm{E}\left(\zeta_{\mathrm{d}}\right)^{*}\right]$, by making use of the classical Higman's result. Finally $U E[G] \cong$ $\left(\times \sum_{d / \mid G_{t} l_{d}} G / G_{t}\right) \times\left(\prod_{d /\left|G_{t}\right|} X_{l_{d}} E\left(\zeta_{d}\right)^{*}\right)$, which finishes the proof in this case.

Let us now assume that $\operatorname{char}(E)=p$ and $G_{p} \neq 1$ (if $G_{p}=1$, the proof is analogous to the above). We may write $\mathrm{G}=\mathrm{G}_{\mathrm{p}} \times \mathrm{M}$ and hence utilizing Lemma 2.2, $\mathrm{UE}[\mathrm{G}]=\mathrm{UE}[\mathrm{M}] \times \mathrm{SE}[\mathrm{G}]$. By the preceding scheme we deduce that

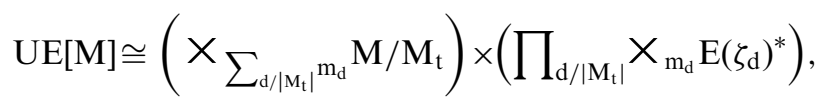

where

$$
\mathrm{m}_{\mathrm{d}}=\mid\left\{\mathrm{g} \in \mathrm{M}_{\mathrm{t}} \mid \mathrm{g} \text { has order } \mathrm{d}\right\} \mid /\left(\mathrm{E}\left(\zeta_{\mathrm{d}}\right): \mathrm{E}\right)
$$

But $M / M_{t} \cong G / G_{t}$ and $M_{t} \cong G_{t} / G_{p}$ since $M_{t} \times G_{p}=G_{t}$, which gives the result immediately. Finally $\mathrm{G}_{\mathrm{p}} \subseteq \mathrm{G}_{\mathrm{t}}$ is bounded, therefore it is not difficult to verify that $\mathrm{SE}[\mathrm{G}]$ is also, by [5]. That is why the Ulm-Kaplansky cardinal functions of SE[G] calculated in $[\mathbf{2 8}, \mathbf{3 5}, \mathbf{3 6}$, completely characterize this group. The proof of (c) is complete.

(d) Trivially by virtue of [36], if $G_{t}$ is finite and char(E) does not divide the cardinality $\left|\mathrm{G}_{\mathrm{t}}\right|$, then $\mathrm{UE}\left[\mathrm{G}_{\mathrm{t}}\right] \cong \prod_{\mathrm{d} /\left|\mathrm{G}_{\mathrm{t}}\right|} \mathrm{X}_{\mathrm{l}_{\mathrm{d}}} \mathrm{E}\left(\zeta_{\mathrm{d}}\right)^{*}$, assuming that char $(\mathrm{E})=0$ or $\operatorname{char}(E)=p>0$ along with $G_{p}=1$ (see also (13)). In view of (13) and (14) we conclude

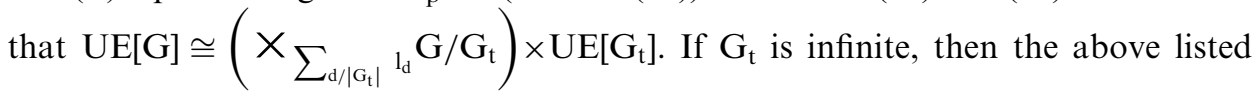
formula (2) from the theorem due to Chatzidakis-Pappas yields (15) in this case.

Now, assume $\operatorname{char}(E)=p \neq 0$ and $G_{p} \neq 1$. Write $G=G_{p} \times M$. From Lemma 2.2 it follows at once that $\mathrm{UE}[\mathrm{G}]=\mathrm{UE}[\mathrm{M}] \times \mathrm{SE}[\mathrm{G}]$. As above,

$$
\mathrm{UE}[\mathrm{M}] \cong\left(\mathrm{X}_{\beta} \mathrm{M} / \mathrm{M}_{\mathrm{t}}\right) \times \mathrm{UE}\left[\mathrm{M}_{\mathrm{t}}\right] \cong\left(\mathrm{X}_{\beta} \mathrm{G} / \mathrm{G}_{\mathrm{t}}\right) \times \mathrm{UE}\left[\mathrm{G}_{\mathrm{t}} / \mathrm{G}_{\mathrm{p}}\right]
$$

because $M / M_{t} \cong G / G_{t}$ and $M_{t} \cong G_{t} / G_{p}$. This completes the proof of (d).

(e) For $G_{t} / G_{p}$ finite, the assertion follows by combining (16) and (12). Let us assume that $G_{t} / G_{p}$ is infinite. Evidently in this situation, (16) and (11) are applicable. Moreover since $G_{p}$ is $\Sigma$-cyclic then, by means of Theorem 2.1(ii) so is SF[G]. 
Therefore the Ulm-Kaplansky invariants computed in $[\mathbf{2 8 , 3 5 , 3 6 ]}$ serve the isomorphism type of $\mathrm{SF}[\mathrm{G}]$. This proves (e).

(f) The first part follows according to (16), (18) and the discussion on the Chatzidakis-Pappas theorem [1, Theorem 3.6]. The second part is a consequence of Claim 2.1 together with $[\mathbf{2 8 , 3 5 , 3 6 ]}$ and [12]. This completes the proof of (f).

REMARK. We now use an example of a group constructed by May [21] to give an assertion pertaining to isomorphism of group algebras over all fields and to the construction of unit groups of such group algebras. Let $\mathrm{G}$ be the abelian group generated by elements $a_{p}, b_{p i}, g_{0}$, and $g_{p j}(p$ prime, $i \geq 1, j \geq 2)$ with relations given in [21]. There it is shown that $G$ is countable and there is no splitting of torsion-free rank one such that $E[G] \cong E\left[G_{t} \times G / G_{t}\right]$, for every field E, so $U E[G] \cong U E\left[G_{t} \times G / G_{t}\right]$. By what we have proved above in Theorem 2.2, the group $U F\left[G_{t} \times G / G_{t}\right]$ is completely determined and so the structure of UF[G] is obviously described.

Remark. When $G$ is arbitrary $\Sigma$-countable (in particular $\Sigma$-cyclic), the formulas (19) and (20) (in particular (17) and (18)) guarantee that the group UF[G] is completely determined up to an isomorphism.

Other significant facts in this area, the reader can see in $[\mathbf{1 6}-\mathbf{1 8} ; \mathbf{2 0}]$.

We conclude this article with some problems of interest and importance that immediately arise.

3. Open questions and conjectures. What is the structure of $\mathrm{UK}[\mathrm{G}]$ when $\mathrm{G}$ is arbitrary (though is not, however, p-splitting)? Probably a formula similar to (11) will be valid. Moreover, what is the simulation for the more general UF[G]? The particular situation when $G_{t} / G_{p}$ is from a class of groups larger than the class of all $\Sigma$-countable groups is also of major interest.

Complying with the structural formulas for $\mathrm{U}[\mathrm{KG}]$ and $\mathrm{UF}[\mathrm{G}]$ listed above, new criteria and computations generalizing those in [29-34] for these groups to belong to some central classes of abelian groups, may be obtained. Nevertheless, this is a problem where some other approach might work.

ACKNOWLEDGEMENTS. Foremost, the author would like to thank the referee whose valuable suggestions considerably improved on the original version of this paper. Also, the author is grateful to Professor Goodearl for his help during the preparation of this work.

\section{REFERENCES}

1. Z. Chatzidakis and P. Pappas, Units in abelian group rings, J. London Math. Soc. (2) 44 (1991), 9-23.

2. P. V. Danchev, Normed unit groups and direct factor problem for commutative modular group algebras, Math. Balkanica 10 (2-3) (1996), 161-173.

3. P. V. Danchev, Commutative group algebras of $\sigma$-summable abelian groups, Proc. Amer. Math. Soc. 125 (9) (1997), 2559-2564.

4. P. V. Danchev, Units in abelian group rings of prime characteristic, Compt. Rend. Acad. Bulg. Sci. 48 (8) (1995), 5-8.

5. P. V. Danchev, Topologically pure and basis subgroups in commutative group rings, Compt. Rend. Acad. Bulg. Sci. 48 (9-10) (1995), 7-10. 
6. P. V. Danchev, Commutative group algebras of direct sums of $\sigma$-summable abelian p-groups, Math. J. Okayama Univ., to appear.

7. P. V. Danchev, Sylow p-subgroups of modular abelian group rings, Compt. Rend. Acad. Bulg. Sci. 54 (2) (2001), 5-8.

8. P. V. Danchev, Isomorphism of commutative modular group algebras, Serdica 23 (23) (1997), 211-224.

9. P. V. Danchev, The splitting problem and the direct factor problem in modular abelian group algebras, Math. Balkanica 14 (3-4) (2000), 217-226.

10. P. V. Danchev, Modular group algebras of coproducts of countable abelian groups, Hokkaido Math. J. 29 (2) (2000), 255-262.

11. P. V. Danchev, Isomorphism of modular group algebras of totally projective abelian groups Comm. Algebra 28 (5) (2000), 2521-2531.

12. L. Fuchs, Abelian groups (Publ. House of the Hungar. Acad. Sci., Budapest, 1958); Infinite abelian groups I (Mir, Moscow, 1974); Infinite abelian groups II (Mir, Moscow, 1977).

13. I. N. Herstein, Noncommutative rings (Mir, Moscow, 1972).

14. G. Karpilovsky, Unit groups of group rings (North-Holland, Amsterdam, 1989).

15. K. Kuratowski and A. Mostowski, Set theory (Mir, Moscow, 1970).

16. W. May, Unit groups of infinite abelian extensions, Proc. Amer. Math. Soc. 25 (1970), 680-683.

17. W. May, Multiplicative groups of fields, Proc. London Math. Soc. (3) 24 (1972), 295-306.

18. W. May, Fields with free multiplicative groups modulo torsion, Rocky Mountain J. Math. 10 (3) (1980), 599-604.

19. W. May, Invariants for commutative group algebras, Illinois J. Math. 15 (1971), 525-531.

20. W. May, Group algebras over finitely generated rings, J. Algebra 39 (1976), 483-511.

21. W. May, Isomorphism of group algebras, J. Algebra 40 (1976), 10-18.

22. W. May, Modular group algebras of totally projective p-primary groups, Proc. Amer. Math Soc. 76 (1) (1979), 31-34.

23. W. May, Modular group algebras of simply presented abelian groups, Proc. Amer. Math. Soc. 104 (2) (1988), 403-409.

24. W. May, The direct factor problem for modular abelian group algebras, Contemp. Math. 93 (1989), 303-308.

25. T. Zh. Mollov, On the multiplicative groups of modular group algebras of primary abelian groups of arbitrary cardinality, Publ. Math. Debrecen 18 (1971), 9-21.

26. T. Zh. Mollov, One remark on the multiplicative groups of semisimple group algebras of abelian p-groups, N. tr. Plovdiv Univ. 20 (1) (1982), 47-52.

27. T. Zh. Mollov, On the multiplicative groups of semisimple group algebras of torsion abelian groups, N. tr. Plovdiv Univ. 20 (1) (1982), 53-67.

28. T. Zh. Mollov, Ulm invariants of Sylow p-subgroups of group algebras of abelian groups over a field of characteristic p, Pliska Stud. Math. Bulgar. 2 (1981), 77-82.

29. T. Zh. Mollov, On the multiplicative groups of semisimple group algebras of abelian p-groups, Compt. Rend. Acad. Bulg. Sci. 35 (12) (1982), 1619-1622.

30. T. Zh. Mollov, On the multiplicative groups of real and rational group algebras of abelian p-groups, Compt. Rend. Acad. Bulg. Sci. 37 (9) (1984), 1151-1153.

31. T. Zh. Mollov, On the invariant pairs of semisimple group algebras of torsion abelian groups, Publ. Math. Debrecen 31 (1984), 145-151.

32. T. Zh. Mollov, Unit groups of semisimple group algebras, Pliska Stud. Math. Bulgar. 8 (1986), 54-64.

33. T. Zh. Mollov and N. A. Nachev, Torsion free rank of the multiplicative group of a commutative semisimple twisted group ring of an abelian group over a field, Compt. Rend. Acad. Bulg. Sci. 43 (5) (1990), 13.

34. T. Zh. Mollov and N. A. Nachev, Torsion free rank of the multiplicative groups of commutative semisimple group algebras over a field, Serdica 16 (2) (1990), 160-165.

35. N. A. Nachev, Invariants of the Sylow p-subgroup of the unit group of a commutative group ring of characteristic p, Compt. Rend. Acad. Bulg. Sci. 47 (1) (1994), 9-12.

36. N. A. Nachev, Invariants of the Sylow p-subgroup of the unit group of a commutative group ring of characteristic p, Comm. Algebra 23 (7) (1995), 2469-2489.

37. S. Perlis and G. L. Walker, Abelian group algebras of finite order, Trans. Amer. Math. Soc. 68 (1950), 420-426. 